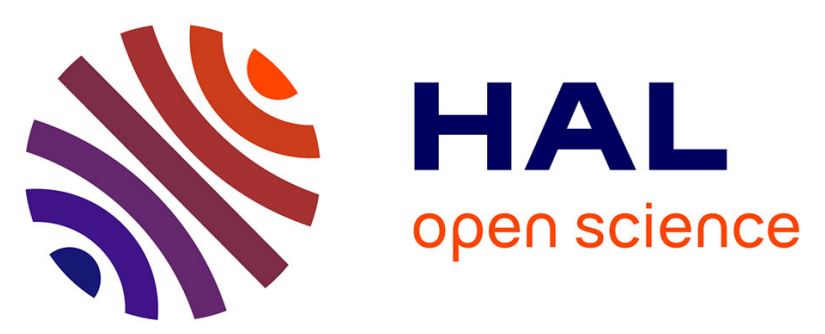

\title{
Multi-Power Irregular Repetition Slotted ALOHA in Heterogeneous IoT networks
}

\author{
Iman Hmedoush, Cédric Adjih, Paul Mühlethaler, Lou Salaun
}

\section{To cite this version:}

Iman Hmedoush, Cédric Adjih, Paul Mühlethaler, Lou Salaun. Multi-Power Irregular Repetition Slotted ALOHA in Heterogeneous IoT networks. PEMWN 2020 - 9th IFIP/IEEE International Conference on Performance Evaluation and Modeling in Wired and Wireless Networks, Dec 2020, Berlin / Virtual, Germany. hal-03043850

\section{HAL Id: hal-03043850 https://hal.science/hal-03043850}

Submitted on 7 Dec 2020

HAL is a multi-disciplinary open access archive for the deposit and dissemination of scientific research documents, whether they are published or not. The documents may come from teaching and research institutions in France or abroad, or from public or private research centers.
L'archive ouverte pluridisciplinaire HAL, est destinée au dépôt et à la diffusion de documents scientifiques de niveau recherche, publiés ou non, émanant des établissements d'enseignement et de recherche français ou étrangers, des laboratoires publics ou privés. 


\section{Multi-Power Irregular Repetition Slotted ALOHA in Heterogeneous IoT networks}

\author{
Iman Hmedoush \\ Inria, France \\ Email: iman.hmedoush@inria.fr
}

\author{
Cédric Adjih, \\ Inria, France \\ Paul Mühlethaler \\ Email: < first name>.<last name>@inria.fr
}

\author{
Lou Salaün \\ Nokia Bell Labs, France \\ Email: lou.salaun@nokia-bell-labs.com
}

\begin{abstract}
Irregular Repetition Slotted Aloha (IRSA) is one candidate member of a family of random access protocols to provide solutions for massive parallel connections in the Internet of Things (IoT) networks. The key features of this protocol are repeating the transmitted packets several times and using Successive Interference Cancellation (SIC) at the decoder to resolve the collisions, which dramatically increases the performance of Slotted ALOHA. Motivated by multiple previous studies of IRSA performance in different settings, we focus on the scenario of an IoT network where the packets of different nodes are received with different powers at the base station, either per design due to different transmission power, or induced by the fact that the nodes are at different distances from the base station. In such a scenario, the capture effect emerges at the receiver, which in turn enhances the protocol performance. We analyze the protocol behavior using a new density evolution which is based on dividing nodes into classes with different powers. By computing the probability to decode a packet in the presence of the interference, we explore the achievable throughput and its associated gain and show the excellent performance of MultiPower IRSA.
\end{abstract}

\section{INTRODUCTION}

\section{A. Modern Random Access Protocols}

A scenario of a typical IoT network is to connect embedded devices, such as environmental sensors, to a central collection node. In many IoT scenarios, there will be a huge inflow of data from devices sharing the same radio spectrum. One of the main challenges of IoT networks is to assign the network resources such that we ensure the connectivity for all devices with a certain rate depending on the required application.

Non-Orthogonal Multiple Access (NOMA) is one of the proposed solutions to solve the issue of massive connectivity in IoT. NOMA often operates at the symbol level, however, an interesting alternative is a family of protocols [1], frequently known as the Coded Slotted Aloha (CSA) family, which has introduced NOMA features to classical ALOHA. They can be considered as modern random access protocols. The common idea of these protocols is to allow each active terminal to send multiple copies of its transmitted packet with the same data and preamble but also to include in each copy an indicator to the positions of the other copies in the frame. On the other side, the receiver will use Successive Interference Cancellation (SIC) to perform an iterative decoding process which removes the successfully decoded packet from all the positions of

This work was partly partly supported by the Inria-Nokia Bell Labs common labs under research action "Network Information Theory". its copies. Contention Resolution Diversity Slotted Aloha (CRDSA) [2] is one variant of CSA family where each packet is repeated only twice, while in Irregular Repetition Slotted Aloha (IRSA) [3], the packet can be repeated a different number of times by each active terminal (i.e. based on a probability distribution function). For the throughput metric, e.g. the number of received packets per slot, it has been shown in [3], that IRSA can achieve a throughput of $T=0.97$ for very large frames (infinite length) and around $T=0.8$ in practical implementations, and [4] proved that $T \rightarrow 1$ with soliton distributions. Previous studies have explored the question of: How can we enhance the performance of IRSA?

One way to increase the achieved throughput of this protocol is through capture: captures occur when two (or more) packets are received with sufficiently different power. The packet with the highest power is recovered first, and then the second after SIC. This paves the way for potentially achieving $T>1$.

\section{B. Capture, related work and our approach Multi-Power IRSA}

In a heterogeneous setting, where IoT nodes are placed in different positions from the base station, the different path loss factors experienced from different channel conditions naturally give rise to the capture effect at the receiver. The effect of such path loss in wireless communication has been extensively studied. By developing stochastic geometry methods, in [5], the received interference and capture probability from a set of nodes in a uniform wireless network is computed. Other studies [6] have considered the case of capture effect with the property of SIC in the receiver. In [7], a study of Irregular Repetition Slotted Aloha (IRSA) in the presence of capture effect and SIC was presented: there, the transmission power is identical for all users and the difference of received power is entirely caused by path loss from distance.

Several schemes where the transmitter power is changed at each transmission have been introduced. In [8], a NOMABased IRSA scheme with different transmission powers is proposed and typical Density Evolution is used for studying the system while the Differential Evolution method is used for optimizing the parameters. In [9], CRDSA with transmit power diversity has been considered. The transmission power distribution was optimized by differential evolution with respect to a total power budget to enhance the throughput.

Our approach called Multi-Power IRSA (MP-IRSA) differs from previous work by introducing the important differences 


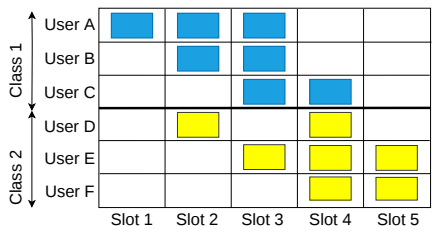

(a) User transmissions on slots

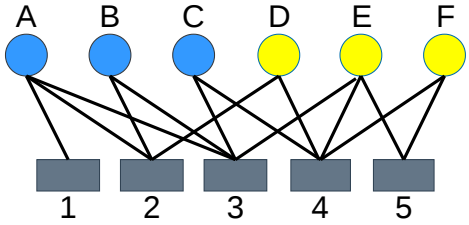

(b) Tanner Graph representation

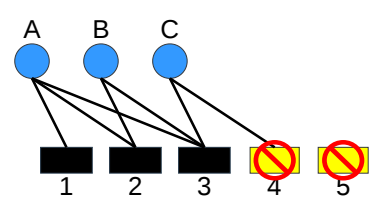

(c) View from Class 1

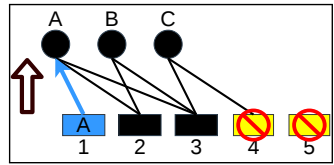

(d) Class 1 step 1

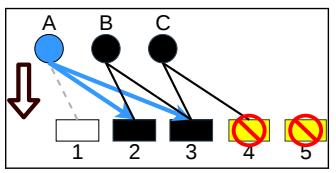

(e) Class 1 step 2

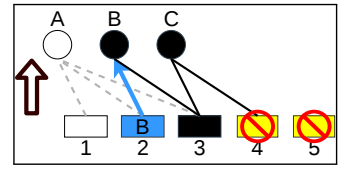

(f) Class 1 step 3

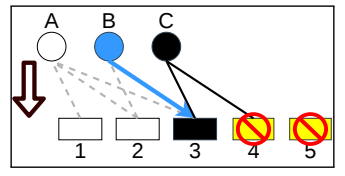

(g) Class 1 step 4

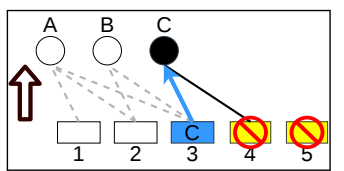

(h) Class 1 step 5

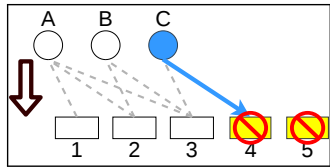

(i) Class 1 step 6

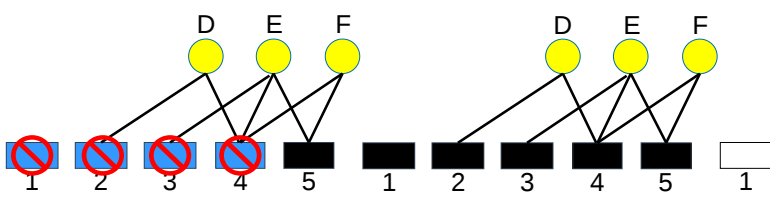

(j) View from Class 2 (k) Class 2 after Class 1 decoding

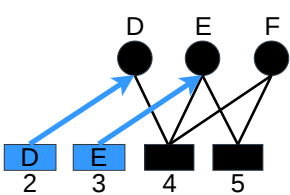

(1) Class 2 step 1

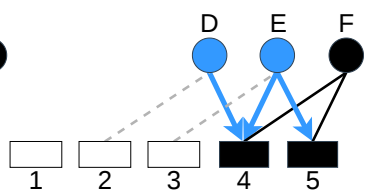

(m) Class 2 step 2

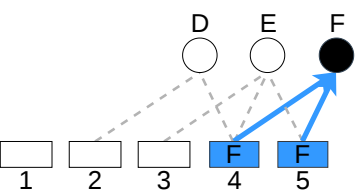

(n) Class 2 step 3

Fig. 1: Example of IRSA, with $\Lambda_{2}=\frac{2}{3}$ and $\Lambda_{3}=13$ - and of the decoding process

that the replicas of the same packet have the same power, and that the users are grouped into classes corresponding to identical received power. MP-IRSA is well-suited to heterogeneous IoT networks, e.g. the signals of farthest terminals arrive at the base station with lower strength, and they might be grouped in classes with lower power: their adjusted transmission power stays low, providing energy savings. MP-IRSA allows the phenomenon of cascading decoding, can increase throughput.

We analyze MP-IRSA through a new variant of multi-class density evolution that relies on the grouping of users with the same received signal power into one class. With that, we are able to study experimentally the performance of the protocol: the influence of each user class on the decoding process and the performance of the other classes are also studied. The impact of the density and the power of the class on the achievable throughput and the maximum load is analyzed.

The paper is organized as follows: Section II introduces the concepts of IRSA with capture and multiple classes. Section III explains the new density evolution of IRSA with multiple classes. Later in section IV, the interference model inside the class is derived. The section $\mathrm{V}$ presents our numerical results and finally, section VI concludes our work.

\section{Principle of IRSA with CAPTURE AND MULTIPLE Classes}

We consider $K$ classes of $N_{c}$ users in a class $c \in K$. Each class contains users whose signals are received with the same power by the base station. Each user has one single packet to transmit (all packets have identical sizes and fit a slot). One considers a frame of size $M$ slots. Each user transmits identical copies of its packet on randomly chosen slots. The number of replicas is picked by each user at the beginning of the frame from a random discrete distribution $\Lambda_{c}$ which is common for all users in our assumption. Precisely, we define:

$\Lambda_{c, i} \triangleq \operatorname{Pr}($ a user of class $c$ repeats his packet $i$ times $)$.
At the end of the frame, the receiver receives the superposition of the physical signals sent by collided users on each slot. We abstract the collision model as: consider a given slot, where $n_{1}+n_{2}+\ldots+n_{k}$ replicas that had been sent from $k$ classes, with $n_{i}$ being the number of users of class $i$ transmitting on that slot. Let us denote the number of replicas of class $i$ that can be recovered in the presence of the interference of other classes as $D_{i}\left(n_{1}, n_{2}, \ldots n_{k}\right)$. Here, we also adopt an intra-class collision model, i.e. whenever $n_{i} \geq 2$, no packet from class $i$ can be recovered on the slot. Packets from other classes, however, might sometimes still be recovered when their received power is higher.

The decoding process is iterative [3], and we consider the following rules while performing the decoding of class $i$ :

- We consider all slots containing exactly one packet from class $i$. In each of these slots, this packet can be recovered if the interference from other classes is sufficiently small (lower than a given threshold).

- Once one packet has been recovered on a certain slot, the copies of this packet can be removed from other slots at the physical signal level by Successive Interference Cancellation (SIC).

A simple example is given in Fig.1a (with Tanner graph in Fig.1b: bipartite graph with users and slots, with one edge for each transmission), and the detailed explanation of the rules of the decoding process is shown in Fig.1. This figure shows two classes with 3 nodes in each class where the received power of class 2 is lower than the received power of class 1. The transmissions from the two classes are colliding on the slots: 2,3 and 4. The decoding process starts with class 1 (Fig.1c) as follows: the intra-class decoding starts first by searching for the packets without collisions (singletons). The packet of user $A$ of class 1 is a singleton received on slot 1 , so it is decoded and removed from its replicas positions on 
slots 2 and 3 as shown in Fig.1d and Fig.1e. The removal of the packet from user $A$ on slot 2 makes the packet of user $B$ on slot 2 colliding only with a replica from user $D$ from class 2. Since class 2 received power is considerably lower than the received power of class 1 , the packet of user $B$ could be considered as a singleton in this case with an "underthreshold" interference from class 2 , and it is in turn decoded and removed from slots 2 and 3 as well as shown in Fig.1f and Fig.1g. Removing the packet of user $B$ from slot 3 makes the packet of user $C$ decodable even in the presence of a replica of user $E$ from class 2 . Note that the packets of class 1 can be removed in the presence of under-threshold interference from class 2 but the opposite is not true. The same process of iterative decoding of class 2 continues (Fig.1k) by removing the packets of users $D$ and $E$ on slots 4 and 5, as shown in Fig.11 and Fig.1m and recovering user $F$ in Fig.1n. Note that in this example, performing the decoding of class 2 packets is impossible before the decoding of class 1 packets (Fig.1j).

In classical IRSA [3], there is only one class. In our work, various decoding order strategies can be adopted depending on when we switch on decoding packets from one class, to decoding packets from another class. When all packets have been retrieved, or no packet from any class can be decoded anymore, the decoding process is considered to be ended. Note that all the strategies will lead to the same final state.

\section{Density Evolution}

The use of the density evolution method for IRSA was pioneered by [3] (and then developed in most of the subliterature devoted to this family of protocols). It models stochastically the decoding process (as in Fig.1), by computing the expected amount of decoded packets at each iteration. It allows us to find the throughput obtained for a given load. We provide a new density evolution formulation for MP-IRSA and use it later for our performance analysis. As for LDPC decoding, the following function is introduced to represent the repetition degree distribution for class $c: \Lambda_{c}(x)=\sum_{i=1}^{l} \Lambda_{c, i} x^{i}$. It is also important to define the edge degree distributions:

where:

$$
\lambda_{c}(x)=\sum_{i=1}^{\ell} \lambda_{c, i} x^{i-1}, \quad \rho(x)=\sum_{i=1}^{\ell} \rho_{i} x^{i-1}
$$

$\lambda_{i, c}$ : defines the probability that a randomly selected edge from the tanner graph in Fig.1b is connected to a user node of class c which has a degree $i$.

$\rho_{i}$ : defines the probability that an edge is connected to a slot node of degree $i$.

This method is a variant of the message passing algorithm [10], where the idea is to perform iterative decoding:

- knowing the average probability that each user's packet has been recovered at the iteration $\ell$, this allows computing the average probability that each slot contains a packet that can be recovered at iteration $\ell$; and conversely,

- knowing the probability that a slot contains a packet that can be recovered at iteration $\ell$, assuming actual decoding followed by SIC, this leads to a new probability that each user's packet is recovered at the end of decoding iteration $\ell$ and the beginning of the iteration $\ell+1$.

Because it is a message passing algorithm, it is more convenient to define, use, and update average probabilities on edges, as done in the LDPC literature. We consider a random edge corresponding to one given user of class $c$ transmitting on a random slot:

- $q_{c, i}$ is the probability that the edge which is connected to one user of class $c$ is not known.

- $p_{c, i}$ is the probability the edge that connects a packet of class $c$ to a random slot is not revealed.

where: $i$ is the iteration number.

The last packet from class $c$ on a slot can be recovered if all the packets from the same class and the classes with a higher power have been already decoded in the previous iterations:

$$
\left(1-p_{c, i}\right)=\delta_{c}\left(1-q_{c, i}\right)^{l-1}
$$

Where: $\delta_{c}$ is the probability that a packet from class $c$ can be decoded in the presence of interference from other classes, and:

$$
\delta_{c}=f_{c}\left(q_{1, i}, q_{2, i}, \ldots . ., q_{c-1, i}\right)
$$

Where $f_{c}$ is the function of the probability of the unknown edges of all classes which will be clearly defined in eq. (7) in the next section. A packet from class $c$ can be recovered if there is at least one copy of this packet that has been decoded on another slot:

$$
q_{c, i}=p_{c, i-1}^{l-1}
$$

Using the polynomial representations of user degree and edge degree distributions, we can average the edge probabilities for class $c$ in (1) and (3) as follows:

$$
p_{c, i}=1-\delta_{c} \cdot \rho\left(1-\lambda_{c}\left(p_{c, i-1}\right)\right)^{l-1}
$$

By letting $M \rightarrow \infty$ (Asymptotic case), we can write:

$$
p_{c, i}=1-\delta_{c} e^{-\frac{G_{c}}{R_{c}} \lambda_{c}\left(p_{c, i-1}\right)}
$$

Where: $G_{c}$ is the load of class $c$ which can be defined as the average number of class $c$ users per slot, i.e, $G_{c}=\frac{N_{c}}{M}$. $R_{c}$ is the rate of class $c$ and is defined as $R_{c} \triangleq \frac{1}{\Lambda_{c}^{\prime}(1)}$. The necessary condition to decode more packets in each class at each decoding iteration is : $p_{c, i}<p_{c, i-1}$ which can be written more precisely using (5) as follows:

$$
1-\delta_{c} e^{-\frac{G_{c}}{R_{c}} \lambda_{c}\left(p_{c}\right)}<p_{c}
$$

Given the parameters $\left(G_{c}, \Lambda_{c}, \delta_{c}\right)$ for each class $c \in K$, one can simply follow the evolution of the decoding process to ultimately estimate the number of decoded packets at the end of the decoding process and also to define the best degree distribution to be given to each class in order to achieve the highest load.

\section{INTER-Class InTERFERENCE MODEL}

In a heterogeneous IRSA setting, where capture effect is considered, the analysis of the decoding process of a packet from a given class $c$ has to take into account two types of packets: the packets from other classes $\forall n \in K$ and $n \neq c$ (considered as interference) that collide on the same slot and 
also collisions from the same-power packets (same class $c$ ). Thus, the density evolution of a class-based IRSA couples two interference models: intra-class interference that can be studied and processed as in classical IRSA in [3] and interclass interference that appears in the factor $\delta_{c}$ for each class. In case of capture at the receiver, when the difference between the strong and the weaker signals is sufficiently large, the weaker signals can be canceled before decoding the strong signal. This gives a condition on the inter-class interference on each slot which affects the decoding process and it can be expressed as:

$$
\delta_{c}=\operatorname{Pr}\left\{\sum_{\substack{n=1 \\ n \neq c}}^{K} X_{n} \Pi_{n} \leq T_{h}\right\}
$$

where: $\Pi_{n}$ is the received power of class $n, X_{n}$ is a random variable representing the number of undecoded packets from class $n$ on the slot, and $T_{h}$ is the interference threshold (SINR) beyond which a packet can be decoded. Indeed, (7) integrates the interference with the effect of path loss and fading on the received power which has been widely covered in wireless networks. Notice that (7) is linked to stochastic geometry [5], in particular when the received power directly depends on distance but also depends on the stage of the decoding process. We can write that $X_{n}$ follows a Poisson distribution with a mean $B_{n}$ which represents the average number of undecoded users from class $n$ on the slot:

$$
B_{n}=\frac{\lambda_{n}\left(q_{n}\right) N_{n} \sum_{l} \Lambda_{n, l} l}{M}
$$

Where: $\lambda_{n}\left(q_{n}\right)$ represents the probability that an edge on class $n$ has not been revealed and $N_{n}$ is the total number of users in class $n$. The sum $\sum_{l} \Lambda_{n, l} l$, represents the average number of replicas of a packet in class $n$. The left side of the inequality in (7) is a sum of scaled Poisson random variables which generally has not a closed formula for its distribution. However, a good approximation can be computed numerically.

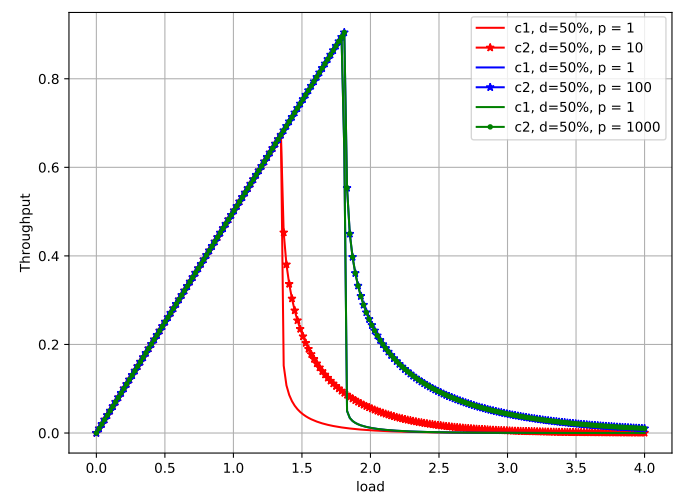

Fig. 2: The throughput of 2 classes with different powers and identical density $50 \%$. Each color is for one scenario with 2 classes.

\section{Numerical Results}

We mainly focus on the asymptotic performance of MPIRSA using our density evolution which is based on classes.

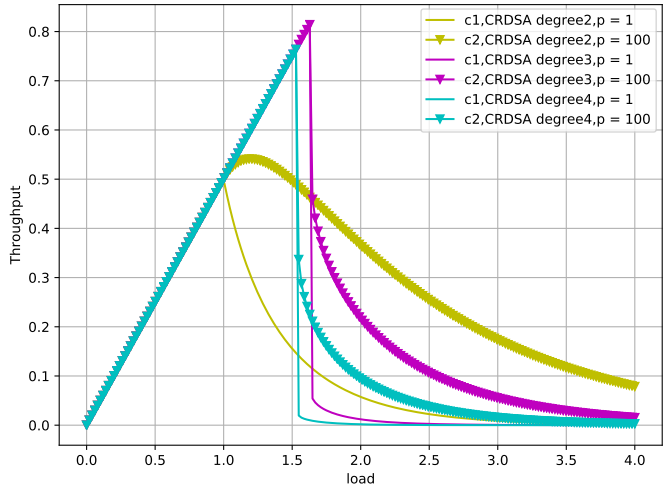

Fig. 3: The throughput of 2 classes of CRDSA with 2, 3 and 4 repetitions with different powers and similar densities

The primary goal is to show that isolating the users with the same received power in a class and taking into account the interference from other classes has a strong and notable impact on the decoding process, since the classes with high received power have more probability to be decoded first, and followed by decoding lower-power classes and so on. Indeed, results evidence a cascading effect on the decoding process starting from the highest-power class to the lowest-power class. Our second objective is to compare the throughput of MP-IRSA with the optimized multi-power CRDSA which was proposed in [9] and also to confirm that it has higher than the throughput of classical IRSA in [3]. This emphasizes the importance of favoring captures into the system design. Our third objective is to explore the impact of the various parameters.

In our numerical experiences, we study the influence of the number of classes, the density of each class (defined as the proportion of the nodes in that class), and the power of each class on the achievable throughput and its associated gain, in different scenarios. The users' degree distribution for IRSA in our analysis is a soliton distribution from [4]. We gradually increase the load ( $\mathrm{x}$-axis of the curves) and plot the corresponding throughput for each class. The total throughput of the network will be the sum of the throughput of all classes.

Fig. 2 shows the throughput achieved by IRSA for 2 equal density classes and different powers. We tested three different cases with a power difference between both classes corresponding to $10 \mathrm{~dB}, 20 \mathrm{~dB}$, and $30 \mathrm{~dB}$ respectively. Typical of IRSA, the curve starts to grow linearly (with near $0 \%$ loss) until a threshold, after what the throughput falls quickly.

For a sufficiently large power difference, i.e. with $P=100$ $(+20 \mathrm{~dB})$ or with $P=1000(+30 \mathrm{~dB})$, the maximum achieved throughput is the near-maximal value: $T_{\max }=0.894$ for the first class and $T_{\max }=0.904$ for the second class. This means that the total throughput is $1.798=0.894+0.904$, a near-doubling of the 1 packet/slot bound without capture. This illustrates from the cascading effect of MP-IRSA with high power difference: the decoder can finishes decoding the packets of the highest power class (intra-class decoding) almost entirely as if the other class did not exist, and then moves to the next class (inter-class decoding).

This phenomenon cannot occur with smaller power differ- 


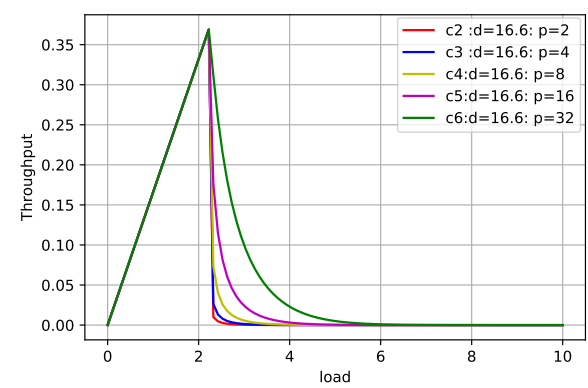

(a) 6 classes with a geometrical difference of 2 in power and SIR = 1

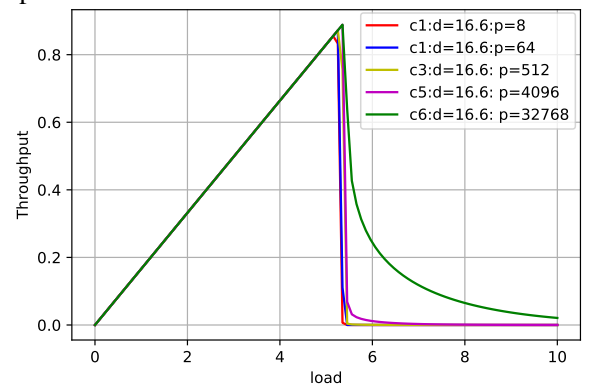

(d) 6 classes with a geometrical difference of 8 in power and SIR $=1$

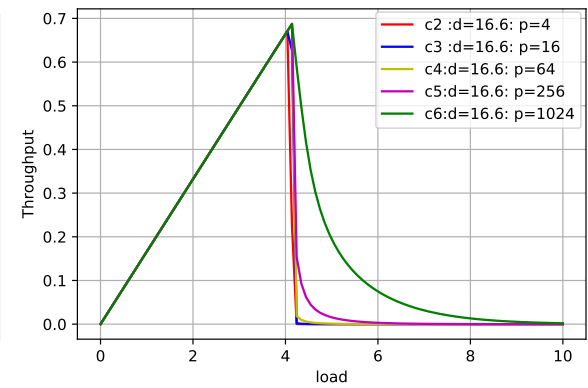

(b) 6 classes with a geometrical difference of 4 in power and SIR = 1

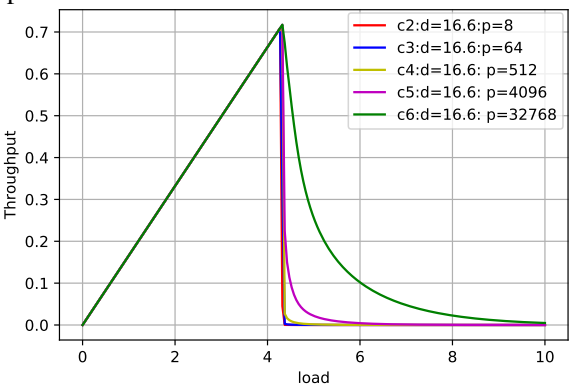

(e) 6 classes with a geometrical difference of 8 in power and SIR $=2$

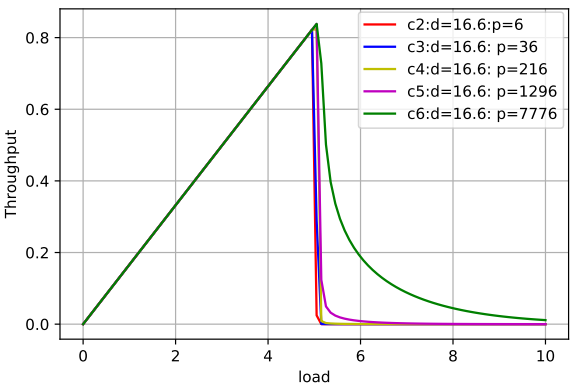

(c) 6 classes with a geometrical difference of 6 in power and SIR $=1$

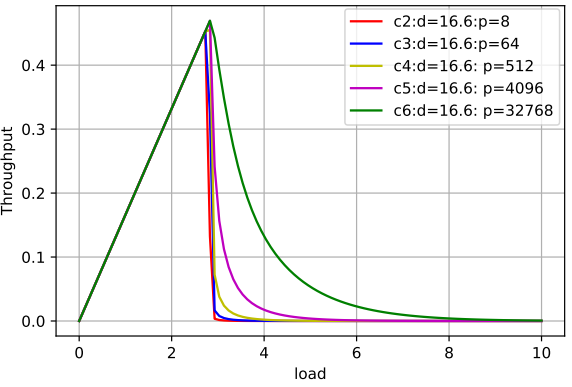

(f) 6 classes with a geometrical difference of 8 in power and SIR $=4$

Fig. 4: The throughput of IRSA with 6 classes and geometrical difference in power and equal densities and SIR = 1

ences, and this is illustrated for $P=10$ : where $T_{\max }=0.673$ for both classes: the decoding process of class 2 can be blocked by class 1 , and the receiver probably will have to decode by cycling back and forth between the classes. The maximum total throughput is 1.346 , significantly less than before.

Fig. 3 shows the performance of 2-classes with CRDSA with 2,3 , and 4 repetitions respectively. The goal of this comparison is to confirm that IRSA can perform better than CRDSA, as in the classical case, as was shown in [3, 4], and confirm this even in the case of classes. With the power of classes set to $(1,100)$, the best degree for CRDSA is 3 as in IRSA without capture. For that value, IRSA indeed still performs better with than CRDSA which achieves a maximum throughput equal to 0.814 . The cascading effect for CRDSA degree 3 and 4 as for IRSA can be guessed.

To understand more the impact of the power difference between the classes on the system performance, we study the case of 6 classes with a geometrical difference in power and for different SIR levels, as in Fig.4. One of the key factors that affect system throughput is users differentiation. As before, when the classes have more difference in power, the obtained throughput can be higher, and this is related to the fact that the created interference from one class to another is more tolerable in the SIC receiver as seen in Fig.4a, Fig.4b, Fig.4c and Fig.4d (for clarity the result of only 5 classes out of 6 are plotted). Interestingly, for a geometric factor of 6 , the total throughput with 6 classes is almost $5(>0.8 \times 6)$. This implies that it is almost if there were 6 cascading decodings, with more than $80 \%$ successfully used slots for each class. For smaller geometric factors, the total throughput is less falls dramatically, and adding more intermediate classes seems to sometimes decrease performances.
Another important key factor is receiver sensitivity (i.e, the SIR). Fig. 4d shows a throughput of $T_{\max }=0.966$ in case of $S I R=1$, while the throughput degrades almost to the half $T_{\max }=0.469$ when the SIR is 4 times greater. This can be seen easily by looking at a packet from the class 3 and one of its interferers in both cases. In case of $S I R=1$, the collision between 8 packets from class 2 and one packet from class 3 can be still decoded since $S I R=\frac{512}{8 \times 64}=1$, While in case of $S I R=4$, the packet of class 3 can be decoded in case of interference with one or two packets at maximum from class 2 which is a degradation by a factor of 4 .

Fig. 5 shows the importance of the number of classes on the achieved throughput and the associated gain. The performance degrades notably when there are more classes in the network as in Fig. $5 \mathrm{c}$ due to the increase in the interference between the classes. The total throughput is around 2.7, 2.0, and 1.6 respectively. It appears as if the intermediate classes were blocking the cascading effect. Thus, the number of classes should be chosen after taking into account different parameters including the received power range and the needed SIR.

Another critical factor is the users' densities in each class. Fig.6 shows different scenarios with different users' densities. Recalling that the decoding process starts from the highest power class, Fig.6a illustrates what occurs when the highest power class has also the highest density. The effect of the class density is not clear in this case: even though the power ratio between classes is 10, lower power classes are often blocking high power one (as in Fig.2), this occurs less often because of their lower density. The impact of class density is clearer in Fig.6b and Fig.6c, where the highest power class (in yellow) is more affected by the interference from the other classes $(87.5 \%$ and $95 \%$ of the users are interferes with class 1$)$. As 


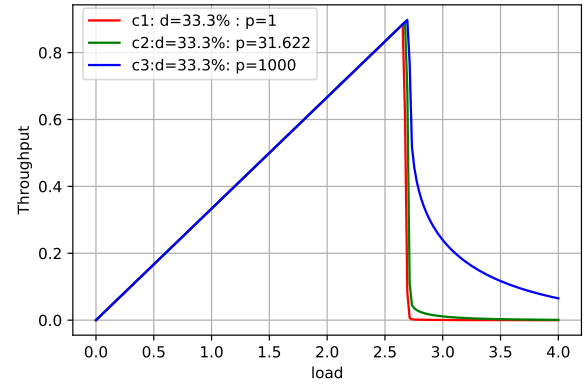

(a) 3 classes in a power range $(0,1000)$

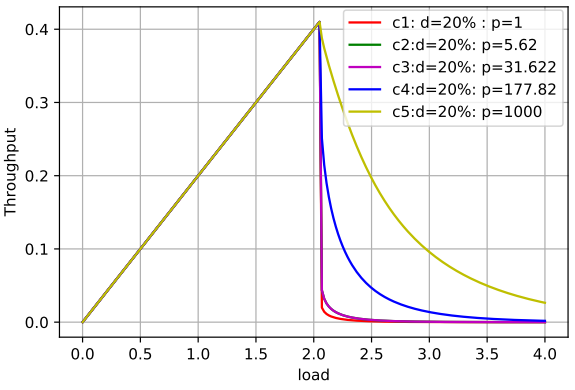

(b) 5 classes in a power range $(0,1000)$

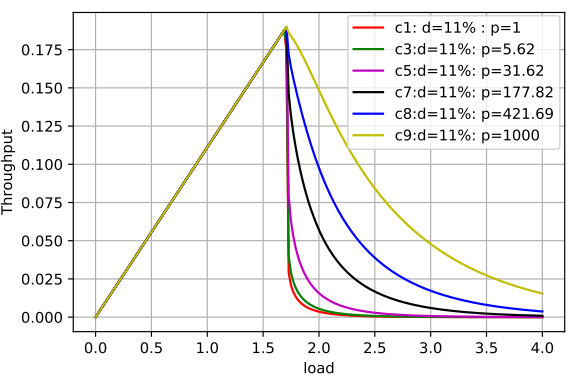

(c) 9 classes in a power range $(0,1000)$

Fig. 5: The effect of increasing the number of classes in the same power range

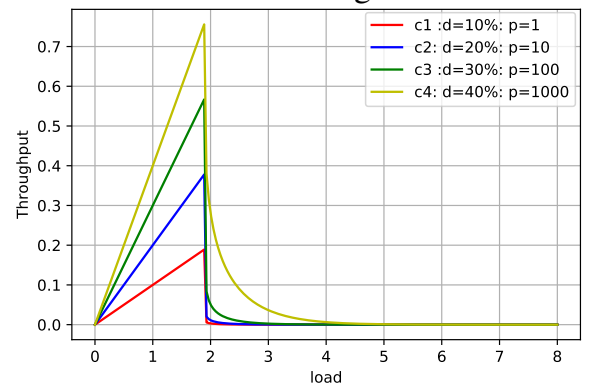

(a) 4 classes with different densities-10,20,30,40

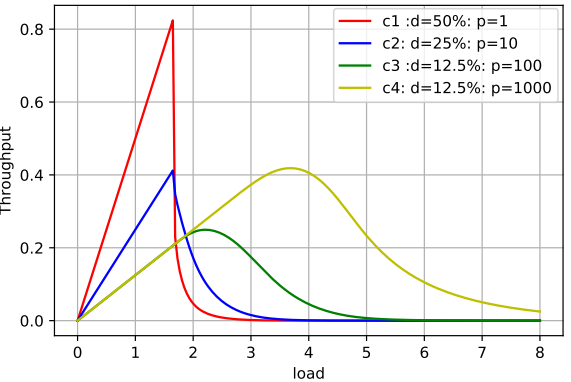

(b) 4 classes with different densities$50,25,12.5,12.5$

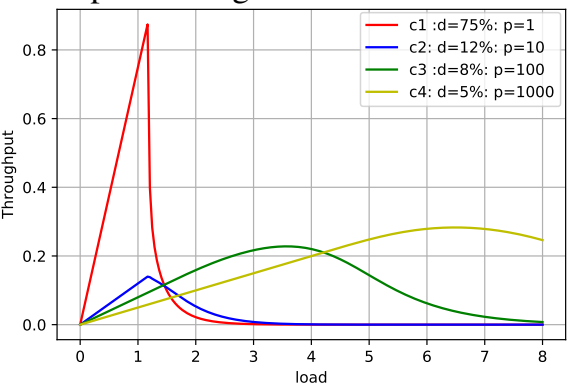

(c) 4 classes with different densities-75,12,8,5

Fig. 6: The effect of different densities of the classes

shown in both figures, the decoding of the highest power class continues correctly (linearly) up to a certain load, and then it degrades slowly affected by the high interference from other high-density classes. Two observations can be made: in those cases, for a higher value of the load, the lower power classes unnecessarily "jam" the channel, because their packet success rate goes to 0 ; but on the other hand, when the degradation of the throughput of higher classes is not reached, the system is interesting as it introduces a form of priority between users.

\section{Conclusion}

In this paper, we formally introduced multi-power IRSA (MP-IRSA), as a random access method, when the replicas of different users are transmitted/received with different powers. We introduced a new density evolution variant based on grouping users into classes: it allows analyzing the performance of MP-IRSA. Multi-power IRSA proves to be a better choice than CRDSA and classical IRSA and confirms the benefits of effects of the capture effect at the receiver. The impact of different system factors on the achieved throughput and the associated load was extensively studied. First, the power difference between the classes and the number of classes plays a huge role in the decoding process, and we observe the best results when the power difference is large: decoding is cascading (one class after the other), instead of cycling (switching between different classes). The receiver sensitivity and the density of each class are other important factors that we studied. Another key factor that we did not explore is the repetition degree distribution: we used the same soliton distribution for all classes. Finding methods to optimize a common degree distribution for all classes or multiple degree distributions for multiple classes, and studying its impact, is a possible future work of this paper.

\section{REFERENCES}

[1] Enrico Paolini, Cedomir Stefanovic, Gianluigi Liva, and Petar Popovski. Coded Random Access: Applying Codes on Graphs to Design Random Access Protocols. IEEE Commun. Mag., 53(6):144-150, 2015.

[2] E Casini, R. D Gaudenzi, and Herrero. O. D. R. Contention resolution diversity slotted aloha (crdsa): An enhanced random access schemefor satellite access packet networks. IEEE Trans. on Wireless Commun., 6(4):1408-1419, 2007.

[3] Gianluigi Liva. Graph-based analysis and optimization of contention resolution diversity slotted aloha. IEEE Transactions on Communications, 59(2):477-487, 2011.

[4] Krishna R Narayanan and Henry D Pfister. Iterative collision resolution for slotted aloha: An optimal uncoordinated transmission policy. In ISTC, Gothenburg, Sweden, 2012.

[5] François Baccelli and Bartlomiej Blaszczyszynz. Stochastic Geometry and Wireless Networks, Volume I - Theory. NoW Publishers,1, 2009.

[6] Xinchen Zhang and Martin Haenggi. The performance of successive interference cancellation in random wireless networks. IEEE Transactions on Information Theory, 60(10):6368 -6388, 2014.

[7] E.E. Khaleghi; C. Adjih; A. Alloum and P. Mühlethaler. Near-far effect on coded slotted aloha. 2017-IEEE 28th Annual International Symposium on Personal, Indoor, and Mobile Radio Communications (PIMRC), pages 1-7, Octobre 2017.

[8] X. Shao; Z. Sun; M. Yang; S. Gu and Q. Guo. Noma-based irregular repetition slotted aloha for satellite networks. IEEE Communications Letters, 23(4):624-627, April 2019.

[9] S. Alvi; S. Durrani and X. Zhou. Enhancing crdsa with transmit power diversity for machine-type communication. IEEE Transactions on Vehicular Technology, 67(8):7790-7794, August 2018.

[10] M. M. Mansour. A turbo-decoding message-passing algorithm for sparse parity-check matrix codes. IEEE Transactions on Signal Processing, 54(11):4376-4392, November 2006. 